\title{
Lift Enhancement for Low-Aspect-Ratio Wings with Periodic Excitation
}

\author{
Kunihiko Taira* and Clarence W. Rowley ${ }^{\dagger}$ \\ Princeton University, Princeton, New Jersey, 08544 \\ Tim Colonius ${ }^{\ddagger}$ \\ California Institute of Technology, Pasadena, California, 91125 \\ David R. Williams ${ }^{\S}$ \\ Illinois Institute of Technology, Chicago, Illinois, 60616
}

Compiled: September 28, 2009

\begin{abstract}
In an effort to enhance lift on low-aspect-ratio rectangular flat-plate wings in low-Reynolds-number post-stall flows, periodic injection of momentum is considered along the trailing edge in this numerical study. The purpose of actuation is not to reattach the flow but to change the dynamics of the wake vortices such that the resulting lift force is increased. Periodic forcing is observed to be effective in increasing lift for various aspect ratios and angles of attack, achieving a similar lift enhancement attained by steady forcing with less momentum input. Through the investigation on the influence of the actuation frequency, it is also found that there exists a frequency at which the flow locks on to a time-periodic high-lift state.
\end{abstract}

\section{Introduction}

As Micro Air Vehicles (MAV) become smaller in size, the Reynolds numbers at which they operate have approached the range where birds and insects fly [1-3]. For example, Black Widow (AeroVironment, Inc. [4]) and Microbat (Caltech [5]) operate around Reynolds numbers of 140, 000 and 20, 000, respectively, while hummingbirds fly around 20,000. At these low Reynolds numbers, the key feature of the flow is the unsteadiness that is generated by the flying body as well as what is inherently in the flow.

Bio-flyers are known to exploit the unsteady aerodynamics for enhanced flight performance. For example, birds flap their wings during take off and flies are observed to achieve increase in lift from the formation of the leading-edge vortices [6-8]. Unsteadiness in the flow also arises from the perturbation in the flight environment, such as wind gusts, obstacles, or other flying objects in the vicinity [3,9]. Thus, understanding the unsteady nature of the flows over low-aspect-ratio wings is an important problem. In fact, Pines and Bohorquez [10] list it as the first area that requires advancement for future development of MAVs.

With unsteady flows around the wings, flow control is an attractive option for stable operations of MAVs. Flow control is also desirable as development of MAVs is considered for agile maneuvers, such as those realized by insects. Loitering, hovering, and perching are some of the desirable maneuvers that MAVs could perform that require new approaches in control techniques as nonlinearity in the flow field would be significant. Thus, novel lift-enhancement approaches are explored in this work, particularly for fixed-wing MAVs.

In the past, a number of flow control studies have been performed. Seifert et al. [11], Greenblatt et al. [12], and Amitay and Glezer [13] have studied the use of periodic excitation for delaying airfoil stall. See Seifert et al. [14] for a review of active separation control. Circulation control that utilizes the Coanda effect to

\footnotetext{
*Postdoctoral Research Associate, Department of Mechanical and Aerospace Engineering. Member AIAA.

${ }^{\dagger}$ Associate Professor, Department of Mechanical and Aerospace Engineering. Associate Fellow AIAA.

$\ddagger$ Professor, Option of Mechanical Engineering. Senior Member AIAA.

$\S$ Professor, Department of Mechanical, Materials, and Aerospace Engineering. Associate Fellow AIAA.
} 
increase spanwise circulation for lift enhancement have also been examined and is reported with a comprehensive coverage by Joslin and Jones [15]. The flow control mechanisms in these past studies have mostly relied on two-dimensionality and have not drawn in the possible aerodynamic benefit from the large-scale three-dimensional effects.

We consider the use of three-dimensional wake vortices and the corresponding vortical forces to enhance aerodynamic performance of low-aspect-ratio wings at post-stall angles of attack. Based on our previous study of the three-dimensional unsteady vortex dynamics around purely translating low-aspect-ratio wings [16], we have recently considered the application of steady blowing for flow control [17]. Unlike most other investigations, the objective was not to reattach the flow or delay separation. The goal of steady actuation was to enhance lift at post-stall angles of attack by modifying the dynamics of the wake vortices, namely the leading-edge, trailing-edge, and tip vortices. As a matter of fact, steady blowing was found to be quite effective in increasing lift (by more than double in many cases) based on a three-dimensional argument for various aspect-ratio wings at post-stall angles of attack.

In a continuing effort to utilize forces generated by the vortices, here we consider the application of a zero-net-mass-flux (ZNMF) actuator, which would require less weight and could be embedded in an MAV more easily than a system necessary for steady blowing. In the current numerical investigation, we mimic the ZNMF actuator by a coarse model of oscillatory momentum injection (external force). The effect of periodic excitation on the wake vortices and the corresponding lift are examined. Comparisons are made with our previous steady flow control study.

\section{Numerical Approach}

\section{Spatial and Temporal Discretization}

In the current study, incompressible viscous flow around a low-aspect-ratio rectangular wing is simulated with the immersed boundary projection method [18]. This method is based upon a second-order staggered-grid finite-volume formulation with Crank-Nicolson and second-order Adams-Bashforth schemes for the viscous and convective terms, respectively. The wing is represented by a set of Lagrangian points, where regularized boundary forces are applied to oppose the flow such that the no-slip condition is satisfied.

Simulations are performed in a box with a dimension of $[-4,6.1] \times[-5,5] \times[-6,6]$ in the $x$ (streamwise), $y$ (vertical), and $z$ (spanwise) directions, where the spatial variables are non-dimensionalized by the chord $c$ of the wing. Grid stretching is applied away from the wing and uniform flow of $\left(U_{\infty}, 0,0\right)$ is applied at the computational boundary except for at the outflow boundary, where a convective boundary condition $\partial \mathbf{u} / \partial t+U_{\infty} \partial \mathbf{u} / \partial x=0$ is specified. Typical grid resolution of $150 \times 66 \times 156$ is used and was verified to be sufficiently converged [16]. The wing is infinitely thin in the limit of $\Delta x \rightarrow 0$ but, with finite resolution, the wing thickness is about one cell-width after discretization due to the use of a regularized delta function (which is the nature of the current immersed boundary method).

The low-aspect-ratio wing is instantaneously materialized at $t=0^{+}$, corresponding to an impulsive translation. It can also be viewed as having potential flow as the initial condition. In this paper, the temporal variable $t$ is taken to be non-dimensionalized by the free stream velocity $U_{\infty}$ and the chord $c$. The time step is chosen such that $U_{\infty} \Delta t / \Delta x \leq 0.5$. For further details on the numerical approach and its validation, see Refs. [16-19].

Throughout this work, we consider a Reynolds number of $R e \equiv U_{\infty} c / \nu=300$, which is above the critical Reynolds number for shedding yet low enough to consider separation in the laminar regime. The lift and drag coefficients are reported as $C_{L} \equiv F_{y} / \frac{1}{2} \rho U_{\infty}^{2} b c$ and $C_{D} \equiv F_{x} / \frac{1}{2} \rho U_{\infty}^{2} b c$, respectively, where $b$ is the wing span. The aspect ratio of the rectangular wing is defined as $A R=b / c$.

\section{Actuator Model}

We consider using a model of the ZNMF actuator [20], also known as the synthetic jet, to modify the dynamics of the wake vortices and the corresponding forces exerted on the wing. This actuator has been found to be more effective in enhancing the aerodynamic characteristics of airfoils than steady blowing or suction $[11,20]$. The current model for the ZNMF actuator is a sinusoidal external force added to the momentum equation:

$$
\mathbf{f}_{\text {act }}=\hat{\mathbf{f}}_{\mathrm{act}}[1-\cos (2 \pi f t)] / 2,
$$


where the blowing direction and magnitude is specified with $\hat{\mathbf{f}}_{\text {act }}$ and the actuation frequency $f$ is prescribed. This forcing function has maximum and minimum magnitudes of $\left|\hat{\mathbf{f}}_{\text {act }}\right|$ and 0 , respectively. Here, we have assumed that the momentum added to the fluid during the suction phase is negligible (i.e., fluid sink). In the present model, no mass is added or subtracted from the continuity equation.

Below we report the control effort with the steady and oscillatory momentum coefficients

$$
\bar{C}_{\mu} \equiv \frac{\rho \bar{u}_{\text {act }}^{2} \sigma b}{\frac{1}{2} U_{\infty}^{2} b c} \quad \text { and } \quad\left\langle C_{\mu}\right\rangle \equiv \frac{\rho\left\langle u_{\text {act }}\right\rangle^{2} \sigma b}{\frac{1}{2} U_{\infty}^{2} b c},
$$

respectively, where the quantities with over bar and \langle\rangle represent time-average and root-mean-square values. The slot width $\sigma$ is set to be the effective width of the regularized delta function [21] that is used to represent the actuator and is $\sigma / c=\Delta x / c=0.04$. To characterize the actuator velocity $u_{\text {act }}$, a simulation is performed with blowing around the wing in quiescent free space with a prescribed force amplitude. The center of forcing is selected for sampling this characteristic velocity.

In the previous steady actuation study [17], we observed that the most effective controller setup is the downstream blowing (tangential to the wing surface) applied along the entire trailing edge as a strip. Hence, we consider the same control setup in the present investigation. The actuator is placed three grid cells $(3 \Delta x / c=0.12)$ above the top surface of the wing to avoid numerical interference between the actuator and the immersed boundary force. A schematic of the actuator setup is presented in Fig. 1.

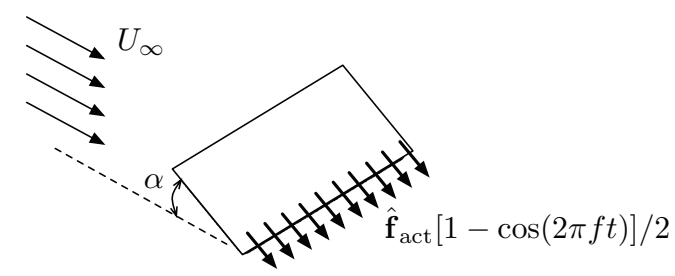

Figure 1. Setup of the trailing-edge actuation.

\section{Post-Stall Flow Control}

When a wing at a post-stall angle of attack undergoes an impulsive translation, the wing experiences high lift due to the creation of the leading-edge vortex and its low-pressure core [16]. The enhancement in lift is lost however when this leading-edge vortex detaches, similar to dynamic stall. A large time after the impulsive start, the lift value is at a substantially lower level. The use of actuation is considered to modify the dynamics of the wake in order to increase lift to the level that is achieved early in time. Our objective is not to delay stall or reattach the flow, but to increase lift by modifying the dynamics of the wake vortices behind the low-aspect-ratio wings at post-stall angles of attack.

It has been observed that steady downstream injection of momentum along the trailing edge is effective in increasing lift [17]. As the actuator applies steady downstream blowing, the trailing-edge vortex sheet is pushed away from the leading-edge vortices and it rolled into the tip vortices (see Fig. 7 in Ref [17]). Such roll up strengthens the tip vortices and increases the downward induced velocity by the tip vortices, which in turn repositions the leading-edge vortices closer to the top surface of the wing generating significant lift increase. For a momentum coefficient of $0.5 \% \leq \bar{C}_{\mu} \leq 1.0 \%$, lift enhancement of 22 to $143 \%$ is attained for wings with various aspect ratios from 1 to 4 , at angles of attack up to $40^{\circ}$.

\section{Application of Periodic Excitation}

Without control, flow around a low-aspect-ratio wing at large time can exhibit a steady state, a periodic shedding, or aperiodic shedding depending on the aspect ratio of the wing and the angle of attack. When the flow is periodic, the dominant features are the shedding of the leading- and trailing-edge vortices at a constant frequency of

$$
S t \equiv f_{n} c \sin \alpha / U_{\infty} \approx 0.12 .
$$

This relation will be extended for angles of attack outside of the periodic states in actuating the flow with blowing. In what follows, we report the frequency non-dimensionalized by the chord and the freestream velocity: $F^{+} \equiv f c / U_{\infty}$. For details on the shedding behavior and the stability of the wake, see Ref. [16]. 
We first consider the application of periodic forcing around a wing of $A R=2$ at $\alpha=30^{\circ}$. Actuation is introduced along the entire trailing edge and directed downstream, parallel to the wing surface. While the current case results in aperiodic flow without control, the excitation frequency is selected by extrapolating the natural shedding frequency for the periodic shedding case (Eq. 3), i.e., $F_{n}=F_{n}^{+} \equiv f_{n} c / U_{\infty}$.

Results with periodic forcing of $\bar{C}_{\mu}=0.5 \%$ and $\left\langle C_{\mu}\right\rangle=0.25 \%$ are presented in Fig. 2 . Shown are the traces of lift and drag coefficients in comparison with cases without control and with steady blowing at $\bar{C}_{\mu}=0.5 \%$ and $1.0 \%$. Here, the maximum forcing magnitude for the periodic forcing case is set to the magnitude of the force in the steady case. From the figure, it can be observed that significant lift increase is similarly achieved with oscillatory actuation as in the steady blowing case. Even without constantly engaging the actuation, we are able to attain lift enhancement at the level between those that are attained by steady momentum injection with $\bar{C}_{\mu}=0.5 \%$ and $1.0 \%$. We note in passing that with control drag is not increased as much as the lift coefficient in comparison to the unactuated case.
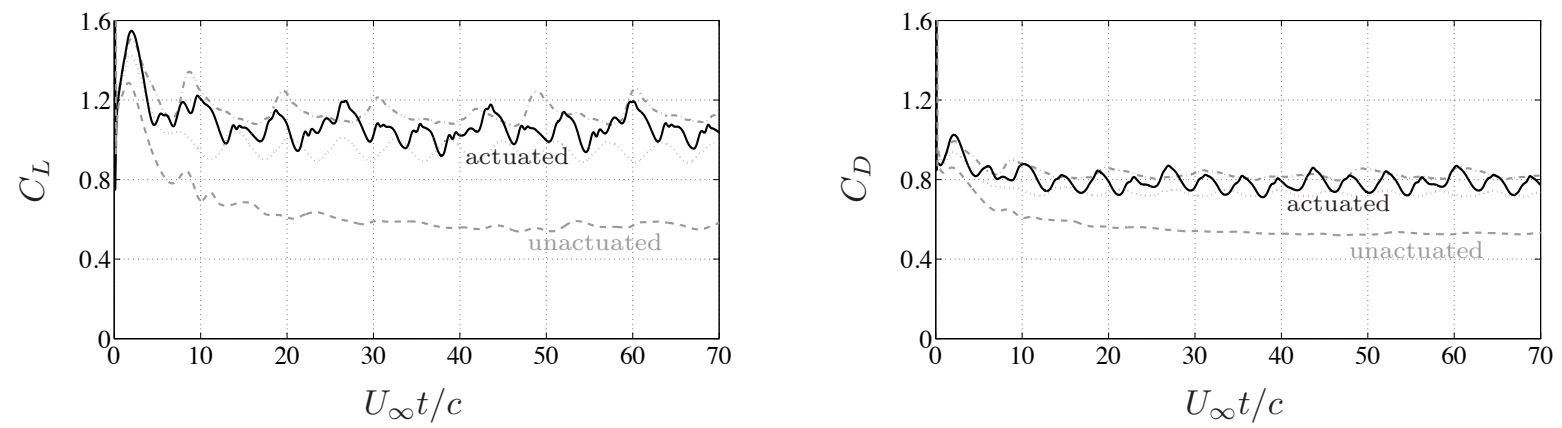

Figure 2. Lift and drag forces on a wing of $A R=2$ at $\alpha=30^{\circ}$ with steady $\left(\cdots \ldots \ldots \ldots . . . \bar{C}_{\mu}=0.5 \%,----\bar{C}_{\mu}=1.0 \%\right)$ and periodic forcing $\left(-, \bar{C}_{\mu}=0.5 \%\right.$ and $\left.\left\langle C_{\mu}\right\rangle=0.25 \%\right)$ along the trailing edge. The case without control is also shown ( - - ).

The corresponding wake structures are visualized in Fig. 3 for cases with (a) no control, (b) steady blowing, and (c) periodic blowing. Shown are the iso-surfaces of $\|\boldsymbol{\omega}\|_{2}=3$ and $Q=2.5$ ( $Q$-criterion [22]), used to capture the vortices and their corresponding cores, respectively. While there are no distinct structures of the tip vortices without actuation, we observe that strong tip vortices are formed with both steady and periodic control. However, the tip vortices with periodic forcing exhibit variation in the strength of the vortex cores in the streamwise direction. By not constantly blowing, the trailing-edge vortex sheet is not always rolled up in the tip vortices. This generates a spanwise variation in the tip vortices. Nonetheless, those tip vortices maintain the self-induced velocity around themselves which presses the leading-edge vortices onto the top surface of the wing for lift enhancement. In fact the vortical structures very close to the wing are almost identical.

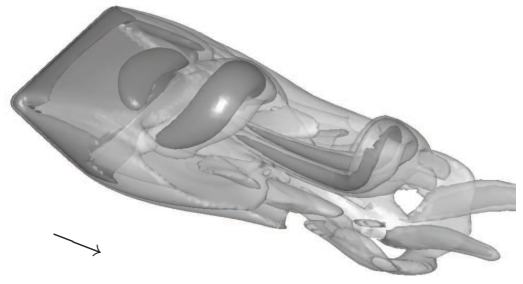

(a) no control

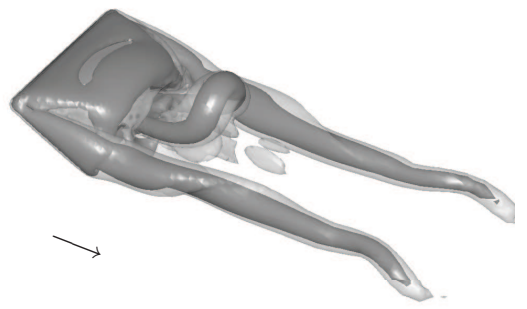

(b) steady control

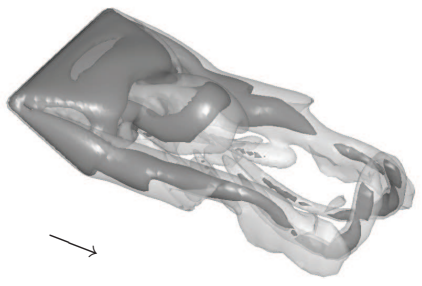

(c) periodic control

Figure 3. Snapshots of the wake structures for cases with and without control. Shown are the iso-surfaces of $\|\omega\|_{2}=3$ (light gray) and $Q=2.5$ (dark gray). Arrows indicate the flow direction.

We further examine whether periodic excitation is effective for wings of other $A R$ at different angles of attack. Shown in Fig. 4 are the time-averaged lift and lift-to-drag ratio at large times. Also shown for comparison are the cases with steady blowing using $\bar{C}_{\mu}=0.5 \%$ and $1.0 \%$. The periodic excitation outperforms the steady blowing for the same time-average forcing effort $\left(\bar{C}_{\mu}=0.5 \%\right)$ in all cases. As in the 
steadily forced cases, our three-dimensional argument for lift enhancement performs well for various lowaspect-ratio wings. Note that the current control setup effectively enhances the aerodynamic performance for a wide range of post-stall angles of attack even at $40^{\circ}$.
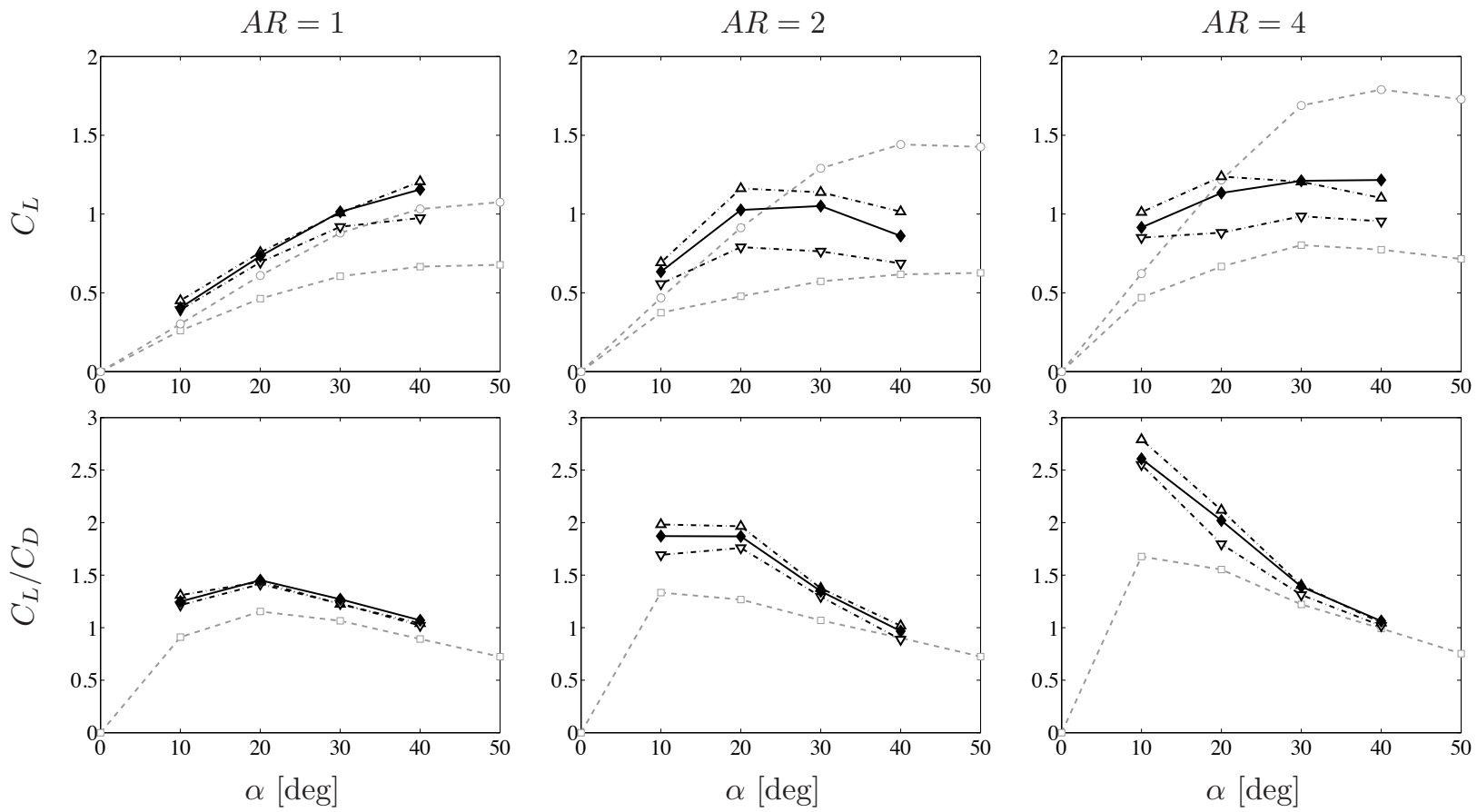

Figure 4. Time-average lift coefficients and lift-to-drag ratios for wings of $A R=1,2$, and 4 . Shown are cases of periodic forcing with $\bar{C}_{\mu}=0.5 \%$ and $\left\langle C_{\mu}\right\rangle=0.25 \%(\diamond)$ as well as cases of steady blowing with $\bar{C}_{\mu}=1 \%(\Delta)$ and $0.51 \%(\nabla)$. Also shown in gray are the unactuated time averaged values $(\square)$ and the maximum lift achieved shortly after the impulsive start $(\circ)$.

\section{Effect of Actuation Frequency}

Next, the actuation frequency is varied from 0.1 to 10 times the natural periodic shedding frequency, $F_{n}^{+}$, around an $A R=2$ wing at $\alpha=30^{\circ}$. The forcing inputs of $\bar{C}_{\mu}=0.5 \%$ and $\left\langle C_{\mu}\right\rangle=0.25 \%$ are selected as in the previous examples. In Fig. 5, the variations in time-average lift are shown for different forcing frequencies. The bars and the shade represent the amplitude of oscillation in the lift coefficient. Also illustrated by the horizontal line is the lift for the uncontrolled case.

For flows excited with frequency above $F_{n}^{+}$, high frequency modulation generated by the forcing frequency is observed. Shown in Fig. 5(a) is the case where $F^{+} / F_{n}^{+}=4$. The overall trends in terms of the average, minimum, and maximum of the lift are similar to the case where the blowing frequency is $F_{n}^{+}$.

One interesting case is observed when we choose to blow the trailing-edge vortex sheet at a frequency slightly less than $F_{n}^{+}$as shown in Fig. $5(\mathrm{~b})$. For $F^{+} / F_{n}^{+}=0.75$, the minimum value of lift is also enhanced, shifting the time-average lift to the largest value out of all frequencies considered here. This agrees with the findings of Seifert et al. [11] that notes the optimal behavior occurs when $F^{+} \approx 1$. As we examine the lift trace, we notice that the there is regular shedding. The difference between this case with other cases is that the roll up of trailing-edge vortex sheet into the tip vortices (strengthening mechanism) and the formation of the leading-edge vortices are in synchronization.

Shown in Fig. 6 are the snapshots of the corresponding wake, sampled at three equally spaced phases $(\phi=0,2 \pi / 3$, and $4 \pi / 3)$ over a shedding period. The first snapshot at $\phi=0$ is selected to be at the time of maximum lift with the leading-edge vortex covering the whole top surface of the wing. As time progresses to $\phi=2 \pi / 3$, the tip vortices start to thin out near the rear corners of the wing with reduced blowing. By the time of $\phi=4 \pi / 3$, the tip vortices cease to roll up the trailing-edge vortex sheet and hence reduces the downward induced velocity applied on the leading-edge vortex. Around this time, the leading-edge vortex sheds from the wing, interestingly in a cleanly shaped vortex ring (clearly visible with the visualization at 


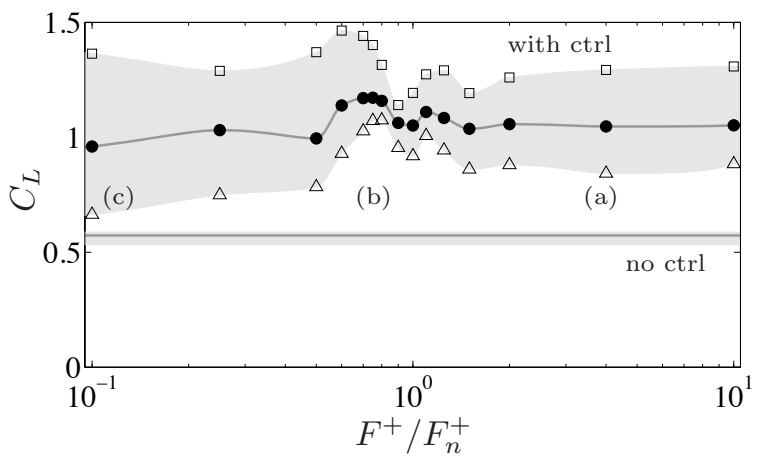

(a)

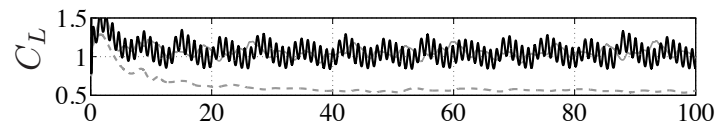

(b)

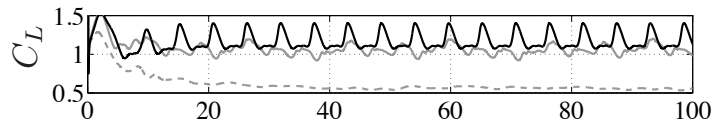

(c)

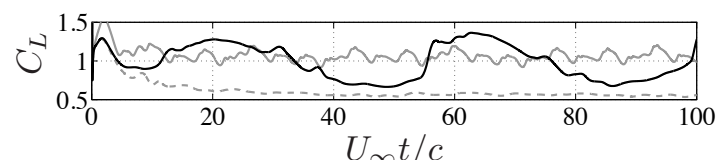

Figure 5. Variation in lift for different actuation frequencies for $A R=2$ and $\alpha=30^{\circ}$ shown on the left. The amplitude of forcing is chosen as $\bar{C}_{\mu}=0.5 \%$ and $\left\langle C_{\mu}\right\rangle=0.25 \%$. Time-average lift coefficients $(\bullet)$ are plotted with corresponding maxima $(\square)$ and minima $(\triangle)$. The horizontal line $(-)$ denotes the average lift for the unactuated case bounded by its minimum and maximum illustrated by the shaded box. Presented on the right are the time history of lift ( - ) for (a) $F^{+} / F_{n}^{+}=4$, (b) $F^{+} / F_{n}^{+}=0.75$, and (c) $F^{+} / F_{n}^{+}=0.1$ with the unactuated and $F^{+} / F_{n}^{+}=1$ results superposed as ( - - ) and ( - ), respectively.

$\phi=2 \pi / 3)$. Due to this synchronization, the flow locks on to a high-lift state by keeping the low-pressure core of the leading-edge vortices as close as possible to the top surface in between their detachment. Note that the actuation and the roll up of the leading-edge vortices are slightly out of phase since there is a time delay required for the trailing-edge vortex sheet to be rolled into the tip vortices and for the leading-edge vortex to grow in size.

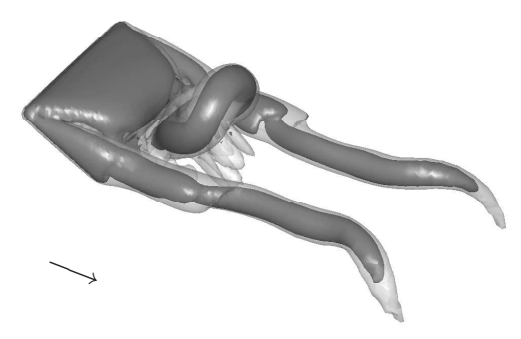

$\phi=0$

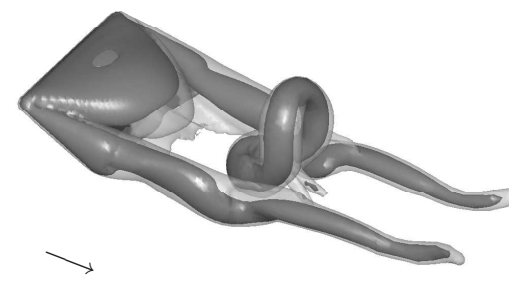

$\phi=2 \pi / 3$

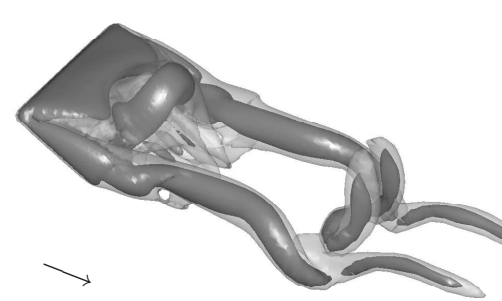

$\phi=4 \pi / 3$

Figure 6. Snapshots of the wake structures shown at one-third phase intervals for the actuated case with $F^{+} / F_{n}^{+}=0.75$. Shown are the iso-surfaces of $\|\boldsymbol{\omega}\|_{2}=3$ (light gray) and $Q=2.5$. Arrows indicate the flow direction.

For forcing frequency of $F^{+} / F_{n}^{+} \lesssim 0.5$, we observe significant variation in lift over time with a decrease in the minimum lift close to the level of the unactuated case. See for example, Fig. $5(\mathrm{c})$, where $F^{+} / F_{n}^{+}=0.1$. The tip vortices convect away from the wing in between the occurrence of blowing, resulting in the loss of the tip vortices' columnar structures and their corresponding downward-induced velocity. The key here is to avoid decrease in lift or its large fluctuation over time, by actuating with a time scale less than the time required for the tip vortices to lose their structures.

While actuation at such low frequency may not be desirable for steady operation, we can view this numerical simulation as a case of applying a burst of blowing on a required basis. It is interesting to note that in this case, lift can be greatly increased from the unactuated level. Although the tip vortices need to be reformed, the unsteady mechanism to achieve a boost in lift smoothly over a short duration of time may be of interest for certain applications, such as perching maneuvers where the course of action can be planned a priori.

We can also regard the present control setup to be robust against perturbations in the flow if one is to consider the actuation frequency as a perturbation in the controller. Thus, the use of this control setup with feedback could possibly stabilize the perturbed flow field in the vicinity of the wing to yield large lift in a robust manner. In a recent work by Ahuja et al. [23], a model-based feedback control is found to 
stabilize the flow about the unstable steady state for analogous two-dimensional flows around a flat wing. A two-dimensional feedback control study by Joe et al. [24] also realized lift increase in a robust manner. Extending the current study by combining such feedback controllers would be a promising path to maintain a state of high lift with suppressed or reduced oscillations.

\section{Conclusion}

In a continuing effort to explore techniques for enhancing lift on low-aspect-ratio wings in low-Reynoldsnumber post-stall flows, we have considered the application of periodic excitation along the trailing edge. Periodic actuation has been found to be effective in enhancing lift by repositioning the leading-edge vortices closer to the top surface of the wing. This benefit has been achieved by strengthening the tip vortices and the corresponding downward-induced velocity exerted on the leading-edge vortices. In comparison to the steadily forced cases, the periodically actuated cases have shorter tip-vortex cores. Nonetheless, these vortices are able to sustain self-induced roll-up of the trailing-edge vortex sheet. The current control setup with periodic excitation is found to be effective in enhancing lift and lift-to-drag ratio for various low-aspect-ratio wings and post-stall angles of attack. In fact, for the use of the same time-average momentum coefficient, the periodic forcing outperforms the steady blowing case. It is also observed that there exists an actuation frequency at which the flow locks on to a high-lift state. Such flow exhibits the synchronization of the growth of the leading-edge vortices and the roll up of the trailing-edge vortex sheet into the tip vortices.

\section{Acknowledgments}

This work was supported by the US Air Force Office of Scientific Research (FA9550-05-1-0369) with some of the computations made possible by the US Department of Defense High Performance Computing Modernization Program.

\section{References}

\footnotetext{
${ }^{1}$ Mueller, T. J., editor, Fixed and flapping wing aerodynamics for micro air vehicle applications, Vol. 195 of Progress in Astronautics and Aeronautics, AIAA, 2001.

${ }^{2}$ Mueller, T. J. and DeLaurier, J. D., "Aerodynamics of small vehicles," Annual Review of Fluid Mechanics, Vol. 35, 2003, pp. $89-111$.

${ }^{3}$ Ho, S., Nassef, H., Pornsinsirirak, N., Tai, Y.-C., and Ho, C.-M., "Unsteady aerodynamics and flow control for flapping wing flyers," Progress in Aerospace Sciences, Vol. 39, 2003, pp. 635681.

${ }^{4}$ Grasmeyer, J. and Keenon, M., "Development of the Black Widow micro air vehicle," 2001, AIAA Paper 2001-012\%.

${ }^{5}$ Pornsin-Sirirak, T. N., Lee, S. W., Nassef, H., Grasmeyer, J., Tai, Y. C., Ho, C. M., and Keenon, M., "MEMS wing technology for a battery-powered ornithopter," Miyazaki, Japan, 2000, The 13th IEEE International MEMS Conference (MEMS 2000).

${ }^{6}$ Dickinson, M. H. and Götz, K. G., "Unsteady aerodynamic performance of model wings at low Reynolds numbers," Journal of Experimental Biology, Vol. 174, 1993, pp. 45-64.

${ }^{7}$ Ellington, C. P., van den Berg, C., Willmott, A. P., and Thomas, A. L. R., "Leading-edge vortices in insect flight," Nature, Vol. 384, 1996, pp. 626-630.

${ }^{8}$ Birch, J. M. and Dickinson, M. H., "Spanwise flow and the attachment of the leading-edge vortex on insect wings," Nature, Vol. 412, 2001, pp. 729-733.

${ }^{9}$ Watkins, S., Milbank, J., Loxton, B. J., and Melbourne, W. H., "Atmospheric winds and their implications for microair vehicles," AIAA Journal, Vol. 44(11), 2006, pp. 2591-2600.

${ }^{10}$ Pines, D. J. and Bohorquez, F., "Challenges facing future micro-air-vehicle development," Journal of Aircraft, Vol. 34(2), 2006, pp. 290-305.

${ }^{11}$ Seifert, A., Darabi, A., and Wygnanski, I., "Delay of airfoil stall by periodic excitation," Journal of Aircraft, Vol. 33(4), 1996, pp. 691-698.

${ }^{12}$ Greenblatt, D., Neuburger, D., and Wygnanski, I., "Dynamic stall control by intermittent periodic excitation," Journal of Aircraft, Vol. 38(1), 2001, pp. 188-190.

${ }^{13}$ Amitay, M. and Glezer, A., "Role of actuation frequency in controlled flow reattachment over a stalled airfoil," $A I A A$ Journal, Vol. 40(2), 2002, pp. 209-216.

${ }^{14}$ Seifert, A., Greenblatt, D., and Wygnanski, I. J., "Active separation control: an overview of Reynolds and Mach numbers effects," Aerospace Science and Technology, Vol. 8, 2004, pp. 569-582.

${ }^{15}$ Joslin, R. D. and Jones, G. S., editors, Applications of Circulation Control Technologies, Vol. 214 of Progress in Astronautics and Aeronautics, AIAA, 2006.

${ }^{16}$ Taira, K. and Colonius, T., "Three-dimensional separated flows around low-aspect-ratio flat plates," Journal of Fluid Mechanics, Vol. 623, 2009, pp. 187-207.

${ }^{17}$ Taira, K. and Colonius, T., "Effect of tip vortices in low-Reynolds-number poststall flow control," AIAA Journal, 2009 in publication.

${ }^{18}$ Taira, K. and Colonius, T., "The immersed boundary method: a projection approach," Journal of Computational Physics, Vol. 225, 2007, pp. 2118-2137.
} 
${ }^{19}$ Colonius, T. and Taira, K., "A fast immersed boundary method using a nullspace approach and multi-domain far-field boundary conditions," Computer Methods in Applied Mechanics and Engineering, Vol. 197, 2008, pp. 2131-2146.

${ }^{20}$ Glezer, A. and Amitay, M., "Synthetic jets," Annual Review of Fluid Mechanics, Vol. 34, 2002, pp. 503-529.

${ }^{21}$ Roma, A. M., Peskin, C. S., and Berger, M. J., "An adaptive version of the immersed boundary method," Journal of Computational Physics, Vol. 153, 1999, pp. 509-534.

${ }_{22}^{2}$ Hunt, J. C. R., Wray, A. A., and Moin, P., "Eddies, stream, and convergence zones in turbulent flows," Tech. Rep. CTR-S88, Center for Turbulence Research, 1988.

${ }^{23}$ Ahuja, S. and Rowley, C. W., "Feedback control of unstable steady states of flow past a flat plate using reduced-order estimators," Journal of Fluid Mechanics, 2009 in review.

${ }^{24}$ Joe, W. T., Taira, K., Colonius, T., MacMynowski, D. G., and Tadmor, G., "Closed-loop control of vortex shedding on a two-dimensional flat-plate airfoil at a low Reynolds number," 2008, AIAA Paper 2008-634. 